\title{
REVIEW ARTICLE Epithelial-to-mesenchymal transition in gallbladder cancer: from clinical evidence to cellular regulatory networks
}

\author{
Sunwang $\mathrm{Xu}^{1}$, Ming Zhan ${ }^{1}$ and Jian Wang
}

Gallbladder cancer (GBC), with late diagnosis, rapid disease progression and early metastasis, is a highly aggressive malignant tumor found worldwide. Patients with GBC have poor survival, low curative resection rates and early recurrence. For such a lethal tumor, uncovering the mechanisms and exploring new strategies to prevent tumor progression and metastasis are critically important. Epithelial-to-mesenchymal transition (EMT) has a prominent role in the early steps of tumor progression and metastasis by initiating polarized epithelial cell transition into motile mesenchymal cells. Accumulating evidence suggests that EMT can be modulated by the cooperation of multiple mechanisms affecting common targets. Signaling pathways, transcriptional and posttranscriptional regulation and epigenetic alterations are involved in the stepwise EMT regulatory network in GBC. Loss of epithelial markers, acquisition of mesenchymal markers and dysregulation of EMT-inducing transcription factors (EMT-TFs) have been observed and are associated with the clinicopathology and prognosis of GBC patients. Therefore, EMT may be a detectable and predictable event for predicting GBC progression and metastasis in the clinic. In this review, we will provide an overview of EMT from the clinical evidence to cellular regulatory networks that have been studied thus far in clinical and basic GBC studies.

Cell Death Discovery (2017) 3, 17069; doi:10.1038/cddiscovery.2017.69; published online 27 November 2017

\section{FACTS}

- EMT markers and EMT-TFs are dysregulated in GBC tumor specimens.

- Multiple mechanisms are involved in EMT and thus regulate GBC tumor progression, including activation/inhibition of specific signaling pathways, transcriptional and posttranscriptional regulation and epigenetic alteration.

- Targeting EMT signaling pathways can be a potential therapeutic strategy for GBC treatment.

\section{OPEN QUESTIONS}

- Can targeting the EMT regulatory network be an effective strategy to achieve GBC growth prohibition or elimination?

- Is it possible to apply EMT markers as an immunohistochemical staining panel for GBC tumorigenesis or invasion ability validation in the clinic?

- How does EMT contribute to traditional chemoresistance in $\mathrm{GBC}$ ?

Gallbladder cancer (GBC) is one of the most aggressive malignant tumors worldwide, and it represents $80-95 \%$ of biliary tract cancers (BTCs) based on autopsy studies and it ranks fifth among the most commonly occurring gastrointestinal cancers. ${ }^{1,2}$ The incidence of $\mathrm{GBC}$ is decreasing because of increased routine cholecystectomy; however, its mortality and prognosis have remained poor. ${ }^{3}$ The overall survival of GBC patients is only 6 months, with 5 -year survival rates of $5-18 \% .^{4,5}$ This high mortality rate is attributable to the rapid progression of the disease and its highly aggressive behavior. Local invasion to the liver or adjacent organs, lymphatic metastasis, peritoneal dissemination and hematogenous metastasis are the main modes of malignant GBC development. ${ }^{6}$ Most patients with GBC are diagnosed at advanced or noncurative stages without surgical indication. ${ }^{4}$ Among patients who undergo curative resection, the recurrence rate remains high, and they typically present with distant recurrence with or without concomitant locoregional recurrence within 12 months after curative resection., ${ }^{7,8}$ For a tumor with this aggressive biological behavior and poor prognosis, it is critical to uncover the mechanisms of GBC progression and metastasis and identify potential therapeutic targets to improve clinical outcomes.

Over the past decades, epithelial-to-mesenchymal transition (EMT) has come to be regarded as a key process for tumor cells to acquire a more malignant phenotype. EMT is a reversible dynamic process that drives polarized epithelial cells to undergo multiple biochemical changes that allow them to gain a motile mesenchymal cell phenotype that loses cell-cell contacts and adhesion capacity. 9,10 Mesenchymal cells can undergo a reverse process termed mesenchymal-to-epithelial transition to restore the epithelial phenotype. EMT contributes to embryonic development and tissue repair, but is also an early metastatic step for tumor cell invasion and migration, and it promotes tumor progression. ${ }^{11}$ Loss of major epithelial markers, such as E-cadherin, and overexpression of mesenchymal markers, including N-cadherin, Vimentin, Fibronectin and S100A4, often occur concomitantly during the EMT process (Figure 1). ${ }^{10,12}$ These dysregulated markers can be tested in tumor specimens via immunohistochemical staining at the protein level or with $\mathrm{qPCR}$ at the transcriptional level, and all are tightly associated with clinical parameters and survival. ${ }^{13}$ There are multiple EMT mechanisms involving different pathways 
Epithelial phenotype

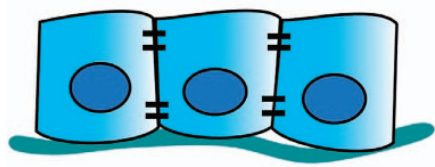

Epithelial markers:

- E-cadherin

- $\beta$-catenin

- Claudin-1

- Occludin
Mesenchymal phenotype

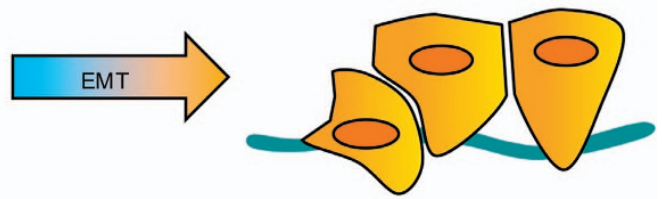

Mesenchymal markers:

- N-cadherin

- Vimentin

- Fibronectin

- s100A4

Figure 1. Cell marker changes in EMT. During EMT, epithelial cells lose their cell membrane epithelial markers and acquire mesenchymal markers and metastatic traits.

and transcription factors, as well as epigenetic alterations, that either promote or suppress tumor development and progression. ${ }^{14,15}$ Through EMT, tumor cells acquire a mesenchymal phenotype and become capable of invading and migrating to local or distant regions, resulting in tumor progression and metastasis.

This review aims to summarize the clinicopathological and prognostic value of EMT markers in GBC patients and present an overview of the cellular regulatory EMT networks associated with GBC progression and metastasis that have been studied thus far. In addition, we provide a preview of current potentially effective chemical agents for targeting EMT.

\section{CLINICAL EVIDENCE OF EMT IN GBC}

Loss of epithelial markers

E-cadherin. E-cadherin, a subtype of the cadherin protein family, is encoded by the $\mathrm{CDH} 1$ gene and is mainly expressed in epithelial cells. As a core component of adherens junctions, E-cadherin has a critical role in mediating and strengthening close membrane apposition between neighboring epithelial cells and participates in the overall polarization of epithelial cells. ${ }^{16}$ Dysregulated or delocalized E-cadherin expression is a hallmark of EMT and has a critical role in tumor cell progression and metastasis. ${ }^{17}$ E-cadherin is localized on the cell membrane of non-tumorous gallbladder epithelial cells, but E-cadherin downregulation or delocalization has been found in GBC tumorous sites via histopathology immunostaining, and a reduction in E-cadherin has been observed in $11.9-70.0 \%$ of GBC specimens (Table 1). ${ }^{18-27}$ The genomic instability of the $C D H 1$ gene, including microsatellite instability and loss of heterozygosity, was found to contribute to the reduced E-cadherin levels in $\mathrm{GBC}^{18}$ Epigenetic silencing through promoter hypermethylation of the $C D H 1$ gene $(11.1-40.9 \%)$ was also observed in GBC tissues, but its relevance in lowering E-cadherin expression is still unclear. ${ }^{28,29}$ In the transition from healthy gallbladder epithelia to inflammatory and tumorous epithelia, downregulated E-cadherin expression is consistently observed. ${ }^{18,19}$ In GBC, it has been confirmed that low E-cadherin expression is correlated with tumor progression and histological differentiation, ${ }^{18,19,25-27}$ pTMN stage and tumor grade, ${ }^{23-27}$ lymph node metastasis, ${ }^{21,25,27}$ and tumor size, ${ }^{20}$ and patients with lower E-cadherin expression exhibit poor survival outcomes. ${ }^{20-22,26,27}$

$\beta$-catenin. Another epithelial EMT marker is $\beta$-catenin, which is abundantly expressed on the cell membrane of non-tumorous specimens, but in tumorous GBC specimens $\beta$-catenin accumulates in the cytoplasm and/or nucleus. ${ }^{30}$ Decreased membranous $\beta$-catenin localization is correlated with tumor progression due to a loss of cell adhesive function. ${ }^{19}$ Increased cytoplasmic $\beta$-catenin accumulation and consequent translocation into the nucleus is often connected with a reduction in the E-cadherin level and altered expression of downstream genes, including genes in the
Wnt signaling cascade and EMT-associated genes. ${ }^{31,32}$ However, the clinical value of the subcellular localization of the endogenous $\beta$-catenin in the cytoplasm and/or nucleus is still controversial. Kimura et al. ${ }^{33}$ and Ghosh et $a l^{34}$ found that cytoplasmic and nuclear $\beta$-catenin accumulation were associated with poor histological differentiation grade and PT stage, but Choi et al. did not find any correlation between clinicopathological factors, overall survival or disease-free survival in GBC patients with cytoplasmic and nuclear $\beta$-catenin accumulation. ${ }^{35}$ Furthermore, Chang et al. ${ }^{36}$ found that cytoplasmic and nuclear $\beta$-catenin accumulation in GBC patients was correlated with less aggressive behavior, and especially cytoplasmic accumulation was associated with improved outcomes. Multiple serine/threonine residues encoded by the third exon of $\beta$-catenin can be phosphorylated by glycogen synthase kinase $3 \beta$, which leads to $\beta$-catenin degradation by the proteasome and prevents $\beta$-catenin cytoplasmic accumulation and nuclear translocation. ${ }^{37}$ Mutations in the $\beta$-catenin phosphorylation region can activate $\beta$-catenin signaling by upholding cellular $\beta$-catenin levels. However, $\beta$-catenin mutations, which were measured as activated mutations in exon three, were rare in GBC patients (ranging from absent to $9.1 \%$ ) and were not correlated with its cellular accumulation. ${ }^{36,38-40}$

Claudin-1. Claudin-1 is a small transmembrane protein that maintains epithelial cell polarity and has a vital role in epithelium homeostasis. ${ }^{41}$ In GBC, membranous Claudin-1 expression was reduced in tumorous sites compared with that in healthy gallbladder epithelium and was associated with aggressive parameters and poor survival. ${ }^{20,42}$

Occludin. Occludin, which acts with Claudin-1 to form intercellular tight junctions on the cytoplasmic membrane, contributes to tight junction stabilization and optimal barrier function in the epithelium. ${ }^{43}$ Loss of Occludin led to failure of epithelial cell tight junctions and is associated with invasion and metastasis in GBC, but patients with higher Occludin expression in tumorous tissue survived longer than those with reduced or no Occludin expression. $^{20}$

\section{Acquisition of mesenchymal markers}

$\mathrm{N}$-cadherin. A switch from $\mathrm{E}$-cadherin to $\mathrm{N}$-cadherin expression is a hallmark of EMT. ${ }^{44} \mathrm{~N}$-cadherin is expressed by mesenchymal cells and is linked to motility and invasion in cancer. There is only one reported study that analyzed $\mathrm{N}$-cadherin expression in GBC samples, and the study confirmed that increased $\mathrm{N}$-cadherin expression was associated with advanced tumor stage, aggressive behavior and poor GBC patient survival. ${ }^{45}$

Vimentin. During EMT, Vimentin upregulation promotes the gain of functional and morphological mesenchymal cell characteristics in epithelial cells and drives the cellular architecture toward a 
Table 1. Clinical relevance of EMT in human GBC

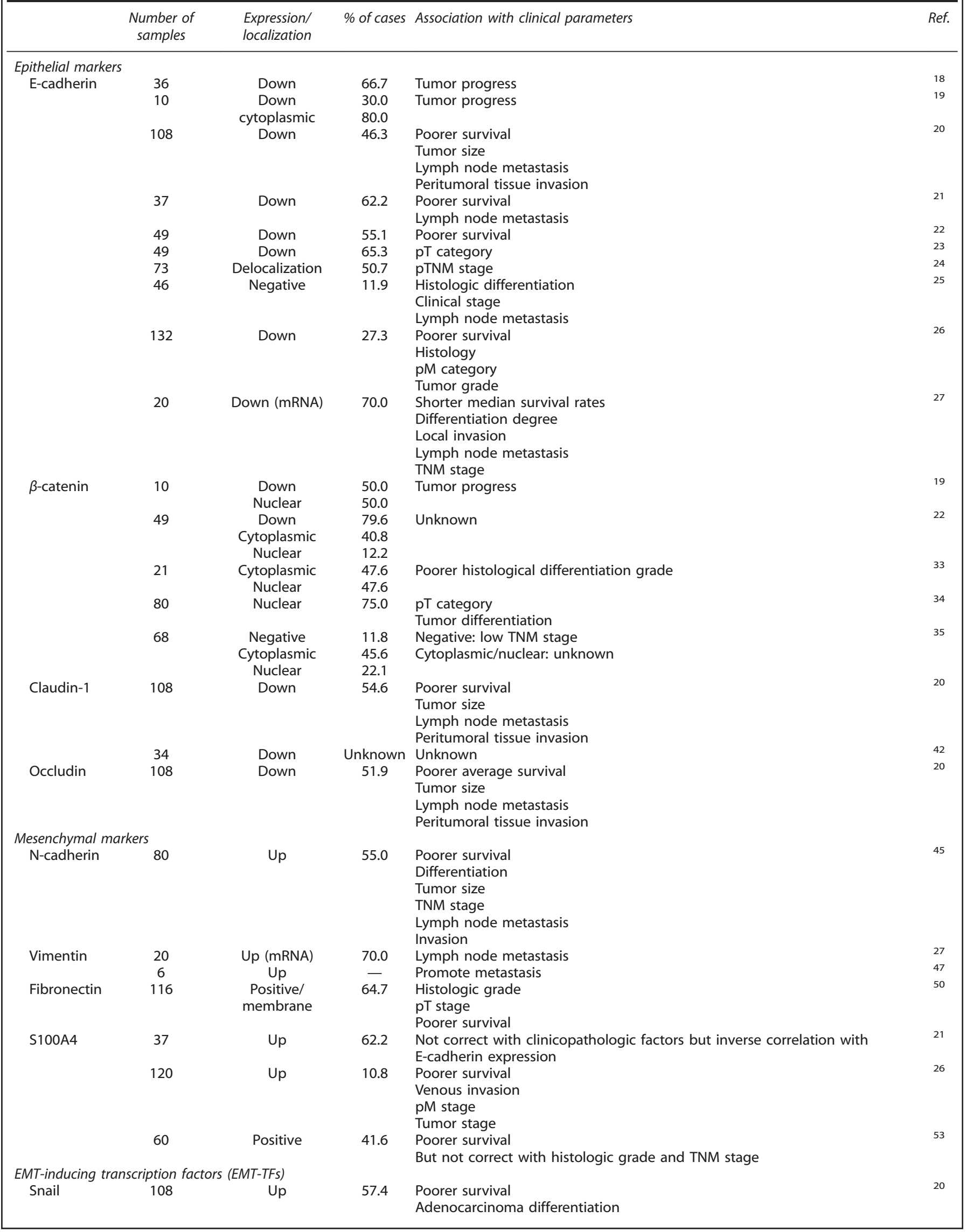


Table 1. (Continued)

\begin{tabular}{|c|c|c|c|c|c|}
\hline & $\begin{array}{l}\text { Number of } \\
\text { samples }\end{array}$ & $\begin{array}{l}\text { Expression/ } \\
\text { localization }\end{array}$ & $\%$ of cases & Association with clinical parameters & Ref. \\
\hline & & & & $\begin{array}{l}\text { Lymph node metastasis } \\
\text { Peritumoral tissue invasion }\end{array}$ & \\
\hline ZEB1 & 30 & Up & 76.7 & Unknown & 57 \\
\hline Twist1 & 20 & Up (mRNA) & 70.0 & $\begin{array}{l}\text { Shorter median survival rates } \\
\text { Differentiation degree } \\
\text { Local invasion } \\
\text { Lymph node metastasis } \\
\text { TNM stage }\end{array}$ & 27 \\
\hline
\end{tabular}

migratory and invasive phenotype. ${ }^{46}$ The Vimentin expression level was increased in GBC specimens and is significantly higher in metastases than in primary tumors, which indicates that Vimentin is associated with GBC metastasis and lymph node metastases. $^{27,47}$

Fibronectin. Fibronectin is a component of the extracellular matrix, which can be expressed and secreted by tumor cells, and excess Fibronectin creates a permissive environment for cancer cell growth and oncogenic progression. ${ }^{48,49}$ Fibronectin also acts as a mesenchymal marker for EMT. Cao et al. ${ }^{50}$ found that Fibronectin expression in GBC tissues was higher than that in the gallbladder epithelium in cholecystitis, and Fibronectin expression in stromal tissues was similar to the levels observed in GBC epithelia. Moreover, Fibronectin expression was significantly associated with histological grade, pT stage and poor survival in GBC patients. ${ }^{50}$

S100 calcium binding protein A4. S100 calcium binding protein A4 (S100A4) promotes EMT by inducing mesenchymal traits in tumors $^{51}$ and is indicative of tumor progression and metastasis. ${ }^{52}$ As a mesenchymal EMT marker, excessive S100A4 expression was inversely correlated with the loss of E-cadherin in $\mathrm{GBC}^{21}$ and was associated with poor GBC patient survival. ${ }^{26,53}$ However, its clinical value with regard to clinicopathological factors is controversial. Chang et al. indicated that S100A4 expression was associated with aggressive GBC phenotypes, ${ }^{26}$ but Kohya et al. and Nakamura et al. found that there was no statistically significant correlation between S100A4 expression and clinicopathological factors in $\mathrm{GBC}^{21,53}$

\section{Dysregulation of EMT-TFs}

EMT is directly orchestrated by several transcription factors (EMTinducing transcription factors (EMT-TFs)), including zinc-finger proteins of the SNAIL superfamily (Snail, Slug and Smuc), zinc finger and E-box-binding proteins of the ZEB family (ZEB1 and ZEB2) and the Twist family of bHLH transcription factors (Twist1 and Twist2). ${ }^{54}$ These EMT-TFs can suppress E-cadherin transcription by directly binding to the E-cadherin promoter and coordinate the inhibition of epithelial genes and activation of mesenchymal genes to initiate EMT and promote cancer progression. ${ }^{55,56}$

Snail is more highly expressed in GBC tissues than in paraneoplastic tissues, and increased Snail expression is associated with histological differentiation, aggressive traits (peritumoral tissue invasion and lymph node metastasis) and poor survival in GBC patients. ${ }^{20}$

ZEB1 expression was also increased in GBC, almost exclusively in the invasive sites but was rarely expressed in non-tumorous epithelia. ${ }^{57}$ Another EMT-TF, Twist1, was more strongly expressed in GBC tissues than in non-tumorous tissues, as measured by both immunohistochemistry staining and mRNA levels in specimens, and ectopic Twist 1 expression was associated with shorter median survival rates, poor differentiation, local invasion and advanced TNM stage. ${ }^{27}$

\section{EMT REGULATORY NETWORKS IN GBC}

Signaling pathways in EMT

Transforming growth factor- $\beta$ signaling pathway. Transforming growth factor- $\beta$ (TGF- $\beta$ ) is one of the most well known and important EMT inducers (Figure 2). ${ }^{58}$ TGF- $\beta$ expression is significantly increased in advanced-stage tumors compared with that in early-stage tumors, and it contributes to angiogenesis and macrophage infiltration in GBC. ${ }^{59}$ In advanced tumors, TGF- $\beta$ promotes tumorigenesis and metastasis by inducing EMT via Smad-dependent and Smad-independent mechanisms. ${ }^{60}$ In GBC cell populations, the abundance of side population cells, which are known as cancer stem cells, was increased by TGF- $\beta$-induced EMT in a Smad3-dependent manner and reduced by withdrawing TGF$\beta$ or silencing Smad3 expression with siRNA. ${ }^{61}$ TGF- $\beta$-induced EMT in GBC cells was accompanied by phosphorylation of PCBP1 (poly $r(C)$-binding protein-1) at serine 43 , but ectopic overexpression of PCBP1 attenuated the $\mathrm{CD}_{4} 4^{+} \mathrm{CD} 24^{-}$stem-cell-like properties induced by TGF- $\beta .^{62}$ With TGF- $\beta$ treatment, expression of genes involved in the oxidation pathway, protein binding and adhesion in GBC cells were primarily altered. ${ }^{63}$ NT5E (etco-5'-nucleotidase, also called (D73), which was the most upregulated gene among 255 dysregulated genes after TGF- $\beta$ treatment, suppressed E-cadherin expression and increased Vimentin expression to promote GBC proliferation and migration in vitro. ${ }^{63}$ TGF- $\beta$ dependent EMT can be inhibited with shRNA to downregulate mTOR levels, resulting in diminished invasion and migration ability in $\mathrm{GBC}^{64}$

The Wnt signaling pathway. The Wnt signaling pathway is required for embryonic development and adult homeostasis, and deregulation of Wht signaling has been implicated in developmental abnormalities and tumor progression. ${ }^{65}$ Activated Wnt signaling prevents $\beta$-catenin degradation by proteasomes followed by $\beta$-catenin nuclear accumulation and interaction with the TCF/LEF (T-cell factor/lymphoid enhancer factor) transcription factor to activate Wnt target gene translation. ${ }^{31,65}$ Sasaki et al. found that epithelial growth factor (EGF) enhanced EMT and stemness acquisition in GBC cells by activating Wnt signaling to induce $\beta$-catenin translocation into the nucleus and repressed the expression of E-cadherin. ${ }^{58}$ Cip7-interacting zinc-finger protein-1 physically interacts with TCF4 to activate $\beta$-catenin/TCF target gene expression, including c-Myc, Snail and Cyclin D1, to promote GBC cell growth and migration. ${ }^{66}$ In contrast, WIF-1 (Wnt inhibitory factor 1), an effective inhibitory factor of the Wnt signaling pathway, targeted $\beta$-catenin to inhibit tumor growth and induce apoptosis of GBC cells. ${ }^{67}$

The Hedgehog signaling pathway. The Hedgehog $(\mathrm{Hh})$ signaling pathway can work alone or can engage in crosstalk with TGF- $\beta$ 


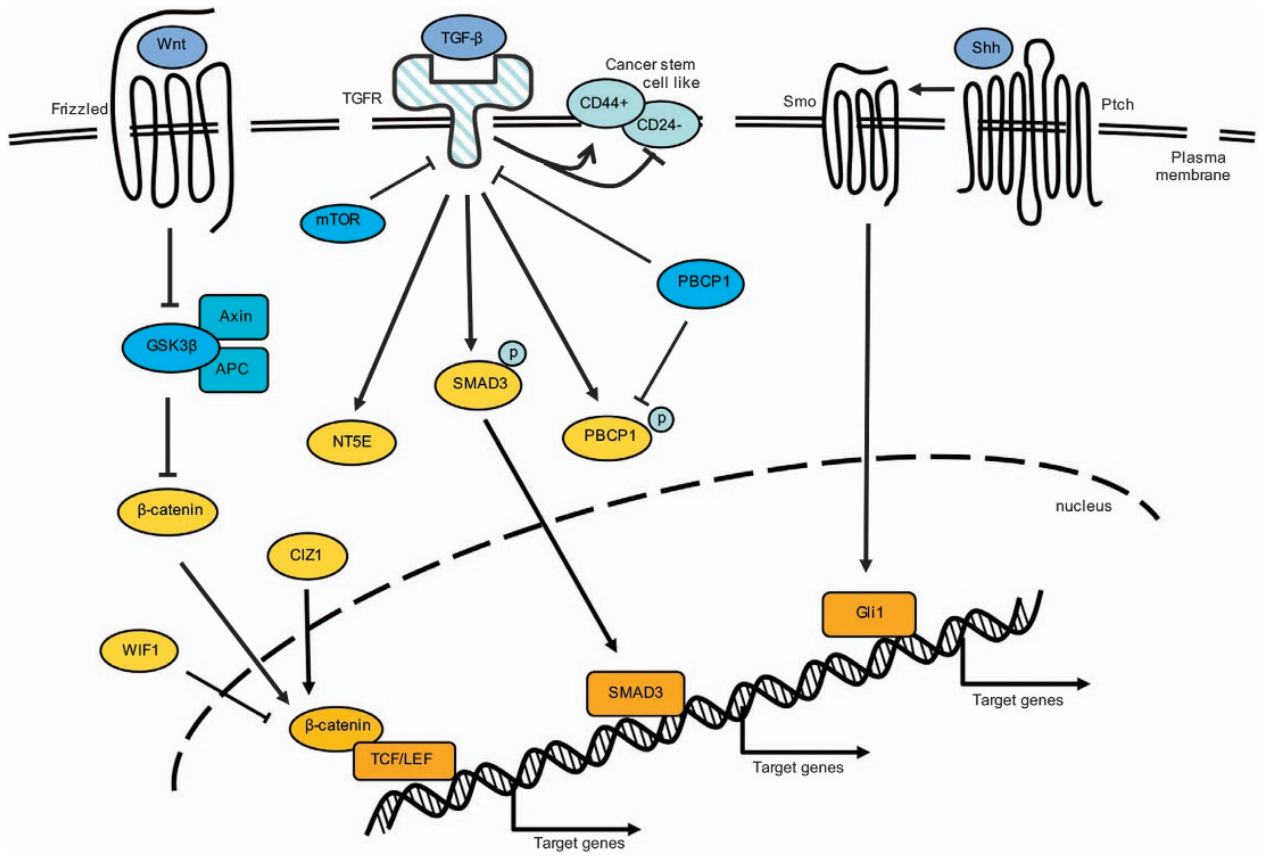

Figure 2. Multiple signaling pathways drive EMT in GBC cells. The TGF- $\beta$ signaling pathway, Wnt signaling pathway and the Hh signaling pathway can govern the switch from an epithelial to a mesenchymal phenotype by upregulating oncogenic or decreasing tumor-suppressive gene expression. In addition, these signaling pathways can be activated or inhibited by multiple cellular factors.

and/or Wnt signaling pathways to initialize and maintain the EMT process. ${ }^{68}$ Three Hh ligands have been identified in the canonical Hh signaling pathway, Sonic Hh (Shh), Indian Hh (Ihh) and Desert $\mathrm{Hh}$ (Dhh). Hh signaling is orchestrated by Patched (Ptch) and Smoothened (Smo), which are both transmembrane receptors. Levels of Shh, its receptor Ptch and the downstream transcription factor Gli1 are frequently increased and significantly associated with tumor stage, lymph node metastasis, venous invasion, hepatic infiltration and poor GBC patient survival. ${ }^{69}$ Specifically, Gli1 was only detectable in the nucleus of tumorous gallbladder cells and was rarely observed in normal gallbladder cells. ${ }^{70}$ The treatment of recombinant human Shh can promote GBC cell proliferation and invasiveness in vitro, but silencing of Smo with siRNA can increase E-cadherin expression by downregulating matrix metalloproteinase (MMP)-3 and MMP-9 expression, resulting in E-cadherin accumulation on the cell membrane followed by a reduction in the percentage of spindle-shaped cells. ${ }^{70}$

\section{Transcription factors in EMT}

Zinc-finger protein SNAI1 (Snail). Snail is the central EMT regulator, and it has been observed to play a role in all EMT processes and is correlated with invasive behavior. ${ }^{71}$ Snail activates EMT by directly modulating epithelial and mesenchymal gene transcription (Figure 3). Snail represses E-cadherin expression by binding to E-box DNA sequences on the E-cadherin promoter region through its carboxy-terminal zinc-finger domain. ${ }^{72}$ Snail expression is controlled by both extracellular growth factors and intracellular networks. Snail has been observed to be activated by exogenous EGF and hepatocyte growth factor stimulation of $\mathrm{GBC}$ cells to induce EMT. ${ }^{58}$ Overexpression of SPOCK1 and TrkB/BDNF in GBC cells enhanced Snail expression and subsequently inhibited E-cadherin expression. ${ }^{73,74}$

Zinc-finger protein SNAI2 (Slug). Slug, another member of the SNAIL zinc-finger protein superfamily, has a role similar to Snail in EMT progression by repressing E-cadherin expression and enhancing Vimentin and Fibronectin expression. ${ }^{71}$ Slug can be positively regulated by MMP-19 through binding to the Slug promoter region, and the subsequent Slug activation increased the expression of the receptor tyrosine kinase Axl to maintain Slug expression via a positive feedback loop and stabilize EMT in GBC cells. $^{75}$

Zinc-finger E-box-binding homeobox 1. Zinc-finger E-box-binding homeobox 1 (ZEB1) is a transcription factor that drives EMT and cancer progression, and its expression often follows activation of Snail expression. ${ }^{72,76}$ ZEB1 was highly expressed in GBC-invasive sites and increased the $\mathrm{GBC}$-invasive potential by repressing $\mathrm{E}$-cadherin and T-cadherin expression and increasing $\mathrm{N}$-cadherin and Vimentin expression at the transcriptional level. ${ }^{57}$ Furthermore, ZEB1 levels were reduced by Forkhead box L1 overexpression to rescue E-cadherin expression and inhibit GBC cell migration and invasion. ${ }^{77}$

JunB. The transcription factor activator protein-1 (AP-1) is one of the major effectors of gene transcription through binding to a consensus DNA sequence in the target gene promoter region. ${ }^{78}$ JunB belongs to the Jun subfamily of the AP-1 family. JunB exerts dual functions in tumors, acting as either an oncogene or tumor suppressor. ${ }^{79}$ However, JunB overexpression was confirmed in $\mathrm{GBC}$ tissues and was related to poor prognosis in $\mathrm{GBC} .^{80}$ JunB expression levels could be enhanced by 3-phosphoinositidedependent protein kinase 1 to decrease E-cadherin expression and maintain the EMT phenotype. ${ }^{80}$

Epigenetic alterations in EMT

Histone modification enzymes in EMT. Lysine-specific demethylase 1A (LSD1), also known as KDM1A (lysine-specific histone demethylase), was the first histone demethylase purified, and it can trigger $\mathrm{H} 3 \mathrm{~K} 4 \mathrm{me} 2$ demethylation to $\mathrm{H} 3 \mathrm{~K} 4 \mathrm{me} 1$ and H3K4me0 ${ }^{81,82}$ LSD1 can be recruited to the E-cadherin promoter by Snail to demethylate H3K4 in the E-cadherin promoter region, resulting in suppressed E-cadherin expression and enhanced cell invasion. ${ }^{83}$ LSD1 can cooperate with c-Myc to induce EMT, leading to GBC cell proliferation and invasion. ${ }^{84}$ LSD1 has been demonstrated to be upregulated in GBC tissues compared with paired 


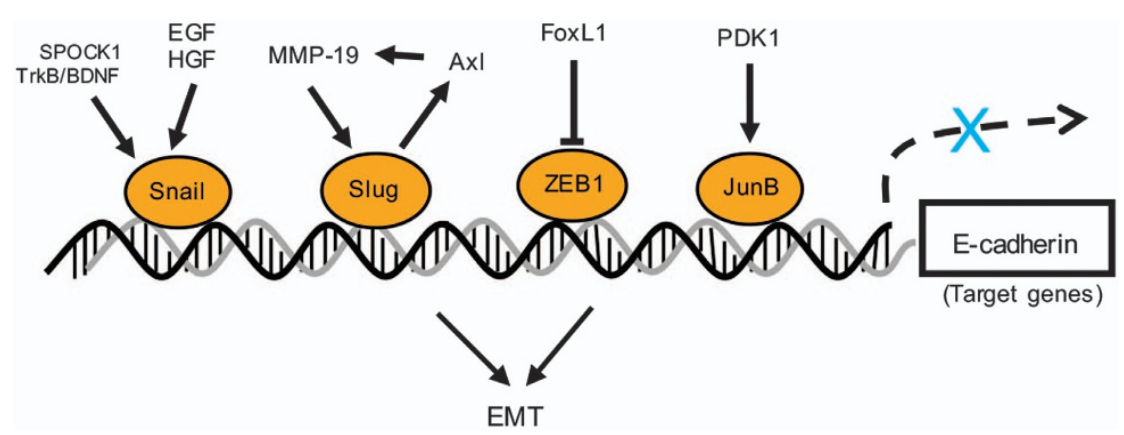

Figure 3. Transcription factor-mediated EMT regulation. EMT-TFs directly bind to their target gene promoter region to regulate target gene transcription and promote tumor cell migration, invasion and metastasis.

normal tissues, and LSD1 upregulation indicates poor outcomes in GBC patients. $^{84}$

Histone acetylation is involved in transcription activation, but deacetylation represses gene transcription. HDAC1, a member of the histone deacetylase (HDAC) family, catalyzes the removal of acetyl residues from histones assembled in inactive regions. ${ }^{85}$ During EMT, HDAC1 is recruited to the E-cadherin promoter by Snail to silence E-cadherin expression via deacetylation of histone $3(\mathrm{H} 3)$ and histone $4 .{ }^{86}$ HDAC1 overexpression can cooperate with TCF-12 to promote EMT-TF (Snail, Slug and Twist2)-mediated transcription and subsequently inhibit E-cadherin transcription, which eventually leads to more invasive and metastatic traits in GBC cells. ${ }^{87}$

\section{Post-transcriptional regulation in EMT}

MicroRNA. MicroRNAs (miRNAs) are short, highly conserved endogenous non-coding RNAs 19-22 nucleotides in length that post-transcriptionally control gene expression by degrading mRNA or inhibiting protein translation through binding to the $3^{\prime}$ untranslated region ( $3^{\prime}$-UTR) of target genes. ${ }^{88}$ miRNAs negatively regulate numerous target genes involved in a variety of pivotal biological processes, such as cell growth, proliferation, differentiation and apoptosis. miRNAs also act as oncogenes or tumor suppressors during tumorigenesis. ${ }^{89-91}$ miRNAs target multiple components involved in epithelial integrity or mesenchymal traits to induce or suppress EMT and metastasis in cancer. In GBC, different sets of miRNAs can work as oncogenes (for example, miR-20a) to promote ${ }^{92,93}$ or tumor suppressors (such as miR-33a, miR-29c-5p, miR-101 and miR-122) to inhibit EMT by regulating their target gene expression (Table 2 ). ${ }^{93-97}$

miR-20a was induced by TGF- $\beta$ in GBC cells and bound to the conserved $3^{\prime}$-UTR of Smad7 to negatively regulate its expression, and the downregulated Smad7 can transactivate miR-20aenhanced $\beta$-catenin reporter gene activity, which resulted in $\beta$ catenin nuclear translocation, a subsequent reduction in E-cadherin expression and an increase in Vimentin expression. ${ }^{92}$ In contrast, miR-29c-5c, miR-101 and miR-122 negatively regulate their target genes, CPEB4, ZFX and PKM2, respectively, and can abrogate TGF- $\beta$-induced EMT and thus act as tumor suppressors to inhibit GBC cell proliferation, invasion and migration. ${ }^{95-97}$ In addition, miR-30a directly binds to Twist mRNA to suppress IL-6induced EMT. ${ }^{94}$ All these oncogenic miRNAs and tumorsuppressive miRNAs are associated with GBC patient clinical outcomes. ${ }^{92,94-96}$

Long non-coding RNA. Long non-coding RNAs (IncRNAs) are a highly heterogeneous group of non-coding RNAs more than 200 nucleotides in length that lack protein-coding potential because of the absence of a discernable open reading frame. IncRNAs can be sorted into one or more of five broad categories: (1) sense, (2) antisense, (3) bidirectional, (4) intronic and (5) intergenic with respect to neighboring coding transcripts. ${ }^{98}$ IncRNAs act in cis or trans to regulate gene expression via multiple distinct molecular mechanisms, including transcriptional and post-transcriptional regulation, modulation of protein stability, acting as competing endogenous microRNA (ceRNA) sponges, modular scaffolding and chromatin remodeling. ${ }^{99,100}$ Emerging evidence has demonstrated that IncRNAs are dysregulated in various cancers and play essential roles in multiple cancer pathogenesis processes, partially by influencing the EMT process. ${ }^{100-102}$ Recently, there have been an increasing number of reports demonstrating that IncRNAs can promote aggressive GBC phenotypes by inducing EMT. $^{103}$

When acting as ceRNAs, IncRNAs can complementarily bind to miRNA to suppress miRNA transcription and restore miRNA target gene expression and function. Thus far, there have been several studies showing that multiple IncRNAs are highly expressed in GBC tissues and activate oncogenes by negatively repressing miRNAs to induce EMT. ${ }^{104-109}$ For example, IncRNA Malat1 directly binds to miR-206 and restores KRAS and ANXA2 (annexin a2) expression, both of which are miR-206 target genes, forming a Malat1/miR-206/KRAS-ANXA2 axis to induce EMT via loss of E-cadherin and acquisition of Vimentin and Twist1 expression. ${ }^{104}$ Other IncRNAs, for example, lincRNA-00152, ${ }^{105}$ IncRNA-H19, ${ }^{107}$ InCRNA-TUG1 ${ }^{108}$ and IncRNA-MINCR, ${ }^{109}$ can bind to miR-138, miR-194-5p, miR-300 and miR-26a-5p, respectively, and silencing IncRNA-H19 and IncRNA-TUG1 in GBC cells reversed TGF- $\beta$ induced EMT. ${ }^{107,108}$ These highly expressed oncogenic IncRNAs were also good indicators of GBC patient clinical outcomes and clinicopathological factors (Table 2). IncRNA-AFP1-AS1, IncRNAROR, IncRNA-KIAA0125 and linc-ITGB1 also act as oncogenic factors to decrease the expression of the epithelial markers E-cadherin or $\beta$-catenin and increase the expression of the mesenchymal markers Vimentin, Twist or Slug to induce EMT in GBC cells, but the underlying molecular mechanisms still needed to be clarified. ${ }^{110-113}$

Pseudogene. Pseudogenes were previously considered nonfunctional genomic relics without protein-coding ability. ${ }^{114,115}$ Recently, increasing evidence has demonstrated that pseudogenes are transcribed into non-coding RNAs that have multilayered biological functions in various cellular processes, especially in cancer. ${ }^{116}$ Several overexpressed pseudogene transcripts have been found to act as EMT inducers in GBC. ${ }^{117,118}$ The NmrA-like family domain containing 1 pseudogene Loc344887 can induce EMT in GBC cells by positively regulating Twist 1 to promote GBC cell proliferation, migration and invasion. ${ }^{117}$ Downregulation of the PTEN mammalian homolog TPTE2 pseudogene, TPTE2P1, inhibited cell migration and invasion capacity by reducing EMT in GBC cells. ${ }^{118}$ 


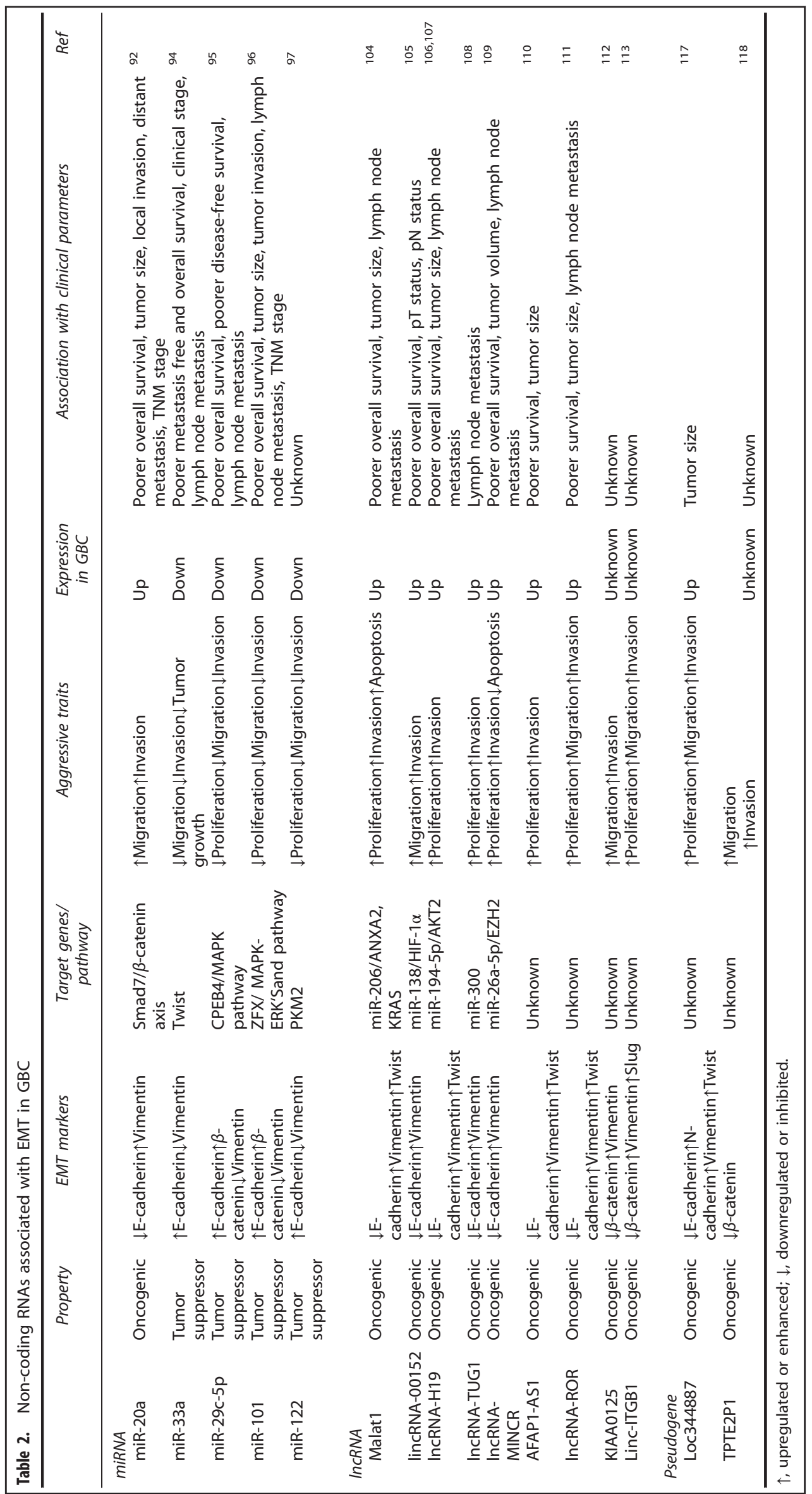


Table 3. Effects of small-molecule inhibitor on EMT in GBC

\begin{tabular}{|c|c|c|c|c|c|}
\hline Inhibitors & Targets & Effects & Mechanisms & Cells & Ref. \\
\hline FH535, DMAT, TBB & Wnt & $\begin{array}{l}\downarrow \text { Viability } \\
\uparrow \text { Apoptosis }\end{array}$ & $\begin{array}{l}\downarrow \text { Wht effectors } \\
\downarrow \text { Cyclin D1 } \\
\uparrow \text { Caspase } 3 / 7 \\
\uparrow p 27\end{array}$ & $\begin{array}{l}\text { MzChA-1 } \\
\text { MzChA-2 } \\
\text { GBC }\end{array}$ & 119 \\
\hline Decitabine & DNA methyltransferase & Unknown & $\begin{array}{l}\downarrow D N A \text { methylation } \\
\uparrow W I F-1\end{array}$ & GBC-SD & 67 \\
\hline SAHA & HDAC & $\downarrow$ Cell growth & $\begin{array}{l}\downarrow \mathrm{HDAC} 1 / 2, \mathrm{EZH} 2 \\
\uparrow \mathrm{E} \text {-cadherin } \\
\uparrow \mathrm{p} 21\end{array}$ & TGBC2TKB & 120 \\
\hline A66 & PI3K p100 $\alpha$ & $\downarrow$ Proliferation & PI3K-AKT effectors & $\begin{array}{l}\text { GBC-SD } \\
\text { NOZ }\end{array}$ & 121 \\
\hline
\end{tabular}

\section{TARGETED EMT THERAPEUTICS IN GBC}

To find potential therapeutic targets, it is critical to uncover the molecular basis and mechanistic properties of EMT. The above review summarized the dysregulated profiles in GBC based on clinical evidence and examination of the cellular regulatory networks associated with EMT. These studies provide potential therapeutic targets for GBC therapy. There are a few well-studied chemical agents that antagonize EMT in GBC (Table 3). Rapamycin is a pharmacological mTOR inhibitor that inhibits EMT by negatively regulating EMT-TFs. Rapamycin diminishes TGF- $\beta$ induced EMT by downregulating ZEB1/2 in a dose-dependent manner in vitro and decreases invasion and migration of GBC in vivo. ${ }^{64}$ Wachter et al. screened five potential anticancer drugs (DMAT, FH535, TBB, myricetin and quercetin) that targeted the Wnt signaling pathway in BTC cells, including three GBC cell lines. ${ }^{119}$ Among them, FH535, DMAT and TBB showed a high cytotoxic effect in all cell lines through inhibition of the Wnt signaling pathway and apoptosis induction. Decitabine (5-aza-2deoxycytidine) might be a candidate drug to eliminate genomic hypermethylation to restore WIF-1 expression levels by inhibiting the Wnt signaling pathway in GBC. ${ }^{67}$ Cyclopamine, a specific $\mathrm{Hh}$ signaling pathway antagonist of Smo that was used for targeting high Smo-expressing tumors, could potentially be an effective inhibitor to suppress GBC cell proliferation and invasion in vitro. ${ }^{70}$ The HDAC inhibitor suberoylanilide hydroxamic acid (SAHA) increased E-cadherin expression and inhibited GBC cell growth by targeting $\mathrm{HDAC} 1 / 2 .{ }^{120}$ Furthermore, the anticancer ability of SAHA was reinforced by silencing EZH2 with siRNA, which indicated that combined therapy with an HDAC inhibitor and an EZH2 inhibitor might be used to inhibit GBC proliferation. Recently, it was found that PIK3CA E545K mutation promoted GBC progression via binding to EGFR followed by downstream Akt activation and EMT promotion. After treatment with the PI3K p110a-selective inhibitor A66, the proliferation rate of GBC cells was significantly reduced both in vitro and in vivo. ${ }^{121}$

\section{FUTURE PERSPECTIVES}

Thus far, there is no doubt that EMT has a pivotal role in $\mathrm{GBC}$ development. Multiple studies have revealed the EMT markers and EMT-TFs that are closely associated with GBC progression, metastasis and prognosis in the clinic, which indicates that EMT can be a detectable and predictable event in GBC progression and metastasis in the clinic. In addition, signaling pathways, transcriptional and post-transcriptional regulation and epigenetic alterations have already been shown to form a comprehensive EMT regulatory network in GBC cells. However, knowledge of other mechanisms, such as the tumor microenvironment or tumor immunology, related to EMT in GBC is still lacking.

An effort to uncover the molecular mechanisms involved in tumor development is essential for us to find effective therapeutic targets to improve clinical outcomes, because the connection between regulatory networks and EMT events provides potentially favorable therapeutic targets for inhibiting GBC progression and metastasis. Further understanding of how to target these EMT processes by applying EMT inhibitors alone or in combination with other drugs is necessary for improving GBC therapeutic strategies.

\section{ACKNOWLEDGEMENTS}

This work was supported by the National Science Foundation of China (no. 81472240 to JW).

\section{COMPETING INTERESTS}

The authors declare no conflict of interest.

\section{PUBLISHER'S NOTE}

Springer Nature remains neutral with regard to jurisdictional claims in published maps and institutional affiliations.

\section{REFERENCES}

1 Lazcano-Ponce EC, Miquel JF, Munoz N, Herrero R, Ferrecio C, Wistuba II et al. Epidemiology and molecular pathology of gallbladder cancer. CA Cancer J Clin 2001; 51: 349-364.

2 Boutros C, Gary M, Baldwin K, Somasundar P. Gallbladder cancer: past, present and an uncertain future. Surg oncol 2012; 21: e183-e191.

3 Bridgewater JA, Goodman KA, Kalyan A, Mulcahy MF. Biliary tract cancer: epidemiology, radiotherapy, and molecular profiling. Am Soc Clin Oncol Educ Book 2016; 35: e194-e203.

4 Hundal R, Shaffer EA. Gallbladder cancer: epidemiology and outcome. Clin Epidemiol 2014; 6: 99-109.

5 Miller KD, Siegel RL, Lin CC, Mariotto AB, Kramer JL, Rowland JH et al. Cancer treatment and survivorship statistics, 2016. CA Cancer J Clin 2016; 66: 271-289.

6 Lai CH, Lau WY. Gallbladder cancer--a comprehensive review. Surgeon 2008; 6 : 101-110.

7 Zhu AX, Hong TS, Hezel AF, Kooby DA. Current management of gallbladder carcinoma. Oncologist 2010; 15: 168-181.

8 Groot Koerkamp B, Fong Y. Outcomes in biliary malignancy. J Surg Oncol 2014; 110: $585-591$. 
9 Kalluri R, Weinberg RA. The basics of epithelial-mesenchymal transition. J Clin Invest 2009; 119: 1420-1428.

10 Zeisberg M, Neilson EG. Biomarkers for epithelial-mesenchymal transitions. J Clin Invest 2009; 119: 1429-1437.

11 Thiery JP, Acloque H, Huang RY, Nieto MA. Epithelial-mesenchymal transitions in development and disease. Cell 2009; 139: 871-890.

12 Tiwari N, Gheldof A, Tatari M, Christofori G. EMT as the ultimate survival mechanism of cancer cells. Semin Cancer Biol 2012; 22: 194-207.

13 Yeung KT, Yang J. Epithelial-mesenchymal transition in tumor metastasis. Mol Oncol 2017; 11: 28-39.

14 Lee JY, Kong G. Roles and epigenetic regulation of epithelial-mesenchymal transition and its transcription factors in cancer initiation and progression. Cell Mol Life Sci 2016; 73: 4643-4660.

15 Nieto MA, Huang RY, Jackson RA, Thiery JP. EMT: 2016. Cell 2016; 166: 21-45.

16 Coopman P, Djiane A. Adherens Junction and E-Cadherin complex regulation by epithelial polarity. Cell Mol Life Sci 2016; 73: 3535-3553.

17 Gumbiner BM. Regulation of cadherin-mediated adhesion in morphogenesis. Nat Rev Mol Cell Biol 2005; 6: 622-634.

18 Priya TP, Kapoor VK, Krishnani N, Agrawal V, Agrawal S. Role of E-cadherin gene in gall bladder cancer and its precursor lesions. Virchows Arch 2010; 456: 507-514.

19 Puhalla H, Herberger B, Soleiman A, Filipits M, Laengle F, Gruenberger T et al. $\mathrm{E}$-cadherin and beta-catenin expression in normal, inflamed and cancerous gallbladder tissue. Anticancer Res 2005; 25: 4249-4254.

20 Xiong L, Wen Y, Miao X, Yang Z. Expressions of cell junction regulatory proteins and their association with clinicopathologic parameters in benign and malignant gallbladder lesions. Am J Med Sci 2011; 342: 388-394.

21 Kohya N, Kitajima Y, Jiao W, Miyazaki K. Effects of E-cadherin transfection on gene expression of a gallbladder carcinoma cell line: repression of MTS1/S100A4 gene expression. Int J Cancer 2003; 104: 44-53.

22 Hirata K, Ajiki T, Okazaki T, Horiuchi H, Fujita T, Kuroda Y. Frequent occurrence of abnormal E-cadherin/beta-catenin protein expression in advanced gallbladder cancers and its association with decreased apoptosis. Oncology 2006; 71: 102-110.

23 Sasatomi E, Tokunaga O, Miyazaki K. Spontaneous apoptosis in gallbladder carcinoma. Relationships with clinicopathologic factors, expression of E-cadherin, bcl-2 protooncogene, and p53 oncosuppressor gene. Cancer 1996; 78: 2101-2110.

24 Kawamoto T, Shoda J, Miyahara N, Suzuki H, Furukawa M, Todoroki $\mathrm{T}$ et al. Expression of MUC1 recognized by a monoclonal antibody MY.1E12 is a useful biomarker for tumor aggressiveness of carcinoma of the gallbladder. Clin Exp Metastasis 2004; 21: 353-362

25 Choi YL, Xuan YH, Shin YK, Chae SW, Kook MC, Sung RH et al. An immunohistochemical study of the expression of adhesion molecules in gallbladder lesions. $J$ Histochem Cytochem 2004; 52: 591-601.

26 Chang HJ, Yoo BC, Kim SW, Lee BL, Kim WH. Significance of PML and p53 protein as molecular prognostic markers of gallbladder carcinomas. Pathol Oncol Res 2007; 13: 326-335.

27 Zhang $M$, Gong W, Zhang Y, Yang Y, Zhou D, Weng $M$ et al. Expression of interleukin- 6 is associated with epithelial-mesenchymal transition and survival rates in gallbladder cancer. Mol Med Rep 2015; 11: 3539-3546.

28 Tozawa T, Tamura G, Honda T, Nawata S, Kimura W, Makino N et al. Promoter hypermethylation of DAP-kinase is associated with poor survival in primary biliary tract carcinoma patients. Cancer Sci 2004; 95: 736-740.

29 Koga Y, Kitajima Y, Miyoshi A, Sato K, Kitahara K, Soejima H et al. Tumor progression through epigenetic gene silencing of $\mathrm{O}(6)$-methylguanine-DNA methyltransferase in human biliary tract cancers. Ann Surg Oncol 2005; 12: 354-363.

30 Moon WS, Park HS, Lee H, Pai R, Tarnawski AS, Kim KR et al. Co-expression of cox2, C-met and beta-catenin in cells forming invasive front of gallbladder cancer. Cancer Res Treat 2005; 37: 171-176.

31 Heuberger J, Birchmeier W. Interplay of cadherin-mediated cell adhesion and canonical Wnt signaling. Cold Spring Harb Perspect Biol 2010; 2: a002915.

32 Valenta T, Hausmann G, Basler K. The many faces and functions of beta-catenin. EMBO J 2012; 31: 2714-2736.

33 Kimura Y, Furuhata T, Mukaiya M, Kihara C, Kawakami M, Okita K et al. Frequent beta-catenin alteration in gallbladder carcinomas. J Ex Clin Cancer Res 2003; 22: 321-328.

34 Ghosh M, Sakhuja P, Singh S, Agarwal AK. p53 and beta-catenin expression in gallbladder tissues and correlation with tumor progression in gallbladder cancer. Saudi J Gastroenterol 2013; 19: 34-39.

35 Choi SY, Jo YS, Huang SM, Liang ZL, Min JK, Hong HJ et al. L1 cell adhesion molecule as a novel independent poor prognostic factor in gallbladder carcinoma. Hum Pathol 2011; 42: 1476-1483.
36 Chang HJ, Jee CD, Kim WH. Mutation and altered expression of beta-catenin during gallbladder carcinogenesis. Am J Surg Pathol 2002; 26: 758-766.

37 Orford K, Crockett C, Jensen JP, Weissman AM, Byers SW. Serine phosphorylation-regulated ubiquitination and degradation of beta-catenin. J Biol Chem 1997; 272: 24735-24738.

38 Kohya N, Kitajima Y, Kitahara K, Miyazaki K. Mutation analysis of K-ras and betacatenin genes related to O6-methylguanin-DNA methyltransferase and mismatch repair protein status in human gallbladder carcinoma. Int J Mol Med 2003; 11: 65-69.

39 Yanagisawa N, Mikami T, Saegusa M, Okayasu I. More frequent beta-catenin exon 3 mutations in gallbladder adenomas than in carcinomas indicate different lineages. Cancer Res 2001; 61: 19-22.

40 Rashid A, Gao YT, Bhakta S, Shen MC, Wang BS, Deng J et al. Beta-catenin mutations in biliary tract cancers: a population-based study in China. Cancer Res 2001; 61: 3406-3409.

41 Tsukita S, Furuse M, Itoh M. Multifunctional strands in tight junctions. Nat Rev Mol Cell Biol 2001; 2: 285-293.

42 Nemeth Z, Szasz AM, Tatrai P, Nemeth J, Gyorffy H, Somoracz A et al. Claudin-1, $-2,-3,-4,-7,-8$, and -10 protein expression in biliary tract cancers. $J$ Histochem Cytochem 2009; 57: 113-121.

43 Cummins PM. Occludin: one protein, many forms. Mol Cell Biol 2012; 32: 242-250.

44 Wheelock MJ, Shintani $Y$, Maeda M, Fukumoto $Y$, Johnson KR. Cadherin switching. J Cell Sci 2008; 121(Pt 6): 727-735.

45 Yi S, Yang ZL, Miao X, Zou Q, Li J, Liang L et al. N-cadherin and P-cadherin are biomarkers for invasion, metastasis, and poor prognosis of gallbladder carcinomas. Pathol ResPract 2014; 210: 363-368.

46 Maier J, Traenkle B, Rothbauer U. Visualizing epithelial-mesenchymal transition using the chromobody technology. Cancer Res 2016; 76: 5592-5596.

47 Dong $\mathrm{P}, \mathrm{He} X \mathrm{~W}, \mathrm{Gu}$ J, Wu WG, Li ML, Yang JH et al. Vimentin significantly promoted gallbladder carcinoma metastasis. Chin Med J 2011; 124: 4236-4244.

48 Williams CM, Engler AJ, Slone RD, Galante LL, Schwarzbauer JE. Fibronectin expression modulates mammary epithelial cell proliferation during acinar differentiation. Cancer Res 2008; 68: 3185-3192.

49 Insua-Rodriguez J, Oskarsson T. The extracellular matrix in breast cancer. Adv Drug Deliv Rev 2016; 97: 41-55.

50 Cao Y, Liu X, Lu W, Chen Y, Wu X, Li M et al. Fibronectin promotes cell proliferation and invasion through mTOR signaling pathway activation in gallbladder cancer. Cancer Lett 2015; 360: 141-150.

51 Xu H, Li M, Zhou Y, Wang F, Li X, Wang L et al. S100A4 participates in epithelialmesenchymal transition in breast cancer via targeting MMP2. Tumor Biol 2016; 37: 2925-2932.

52 Mishra SK, Siddique HR, Saleem M. S100A4 calcium-binding protein is key player in tumor progression and metastasis: preclinical and clinical evidence. Cancer Metastasis Rev 2012; 31: 163-172.

53 Nakamura T, Ajiki T, Murao S, Kamigaki T, Maeda S, Ku Y et al. Prognostic significance of S100A4 expression in gallbladder cancer. Int J Oncol 2002; 20: 937-941.

54 Puisieux A, Brabletz T, Caramel J. Oncogenic roles of EMT-inducing transcription factors. Nat Cell Biol 2014; 16: 488-494.

55 Sanchez-Tillo E, Liu Y, de Barrios O, Siles L, Fanlo L, Cuatrecasas M et al. EMTactivating transcription factors in cancer: beyond EMT and tumor invasiveness. Cell Mol Life Sci 2012; 69: 3429-3456.

56 De Craene B, Berx G. Regulatory networks defining EMT during cancer initiation and progression. Nat Rev Cancer 2013; 13: 97-110.

57 Adachi Y, Takeuchi T, Nagayama T, Ohtsuki Y, Furihata M. Zeb1-mediated T-cadherin repression increases the invasive potential of gallbladder cancer. FEBS Lett 2009; 583: 430-436.

58 Sasaki T, Kuniyasu H, Luo Y, Kato D, Shinya S, Fujii K et al. Significance of epithelial growth factor in the epithelial-mesenchymal transition of human gallbladder cancer cells. Cancer Sci 2012; 103: 1165-1171.

59 Kitamura K, Kasuya K, Tsuchida A, Mimuro A, Inoue K, Aoki T et al. Immunohistochemical analysis of transforming growth factor beta in gallbladder cancer. Oncol Rep 2003; 10: 327-332.

60 Zhang YE. Non-Smad signaling pathways of the TGF-beta family. Cold Spring Harb Perspect Biol 2017; 9: a022129.

61 Zhang Z, Zhu F, Xiao L, Wang M, Tian R, Shi C et al. Side population cells in human gallbladder cancer cell line GBC-SD regulated by TGF-beta-induced epithelial-mesenchymal transition. J Huazhong Uni Sci Technol Med Sci 2011; 31: 749-755.

62 Zhang HY, Dou KF. PCBP1 is an important mediator of TGF-beta-induced epithelial to mesenchymal transition in gall bladder cancer cell line GBC-SD. Mol Biol Rep 2014; 41: 5519-5524. 
63 Xiong L, Wen Y, Miao X, Yang Z. NT5E and FcGBP as key regulators of TGF-1induced epithelial-mesenchymal transition (EMT) are associated with tumor progression and survival of patients with gallbladder cancer. Cell Tissue Res 2014; 355: 365-374.

64 Zong H, Yin B, Zhou H, Cai D, Ma B, Xiang Y. Inhibition of mTOR pathway attenuates migration and invasion of gallbladder cancer via EMT inhibition. Mol Biol Rep 2014; 41: 4507-4512.

65 Nusse R, Fuerer C, Ching W, Harnish K, Logan C, Zeng A et al. Wnt signaling and stem cell control. Cold Spring Harb Symp Quant Biol 2008; 73: 59-66.

66 Zhang D, Wang Y, Dai Y, Wang J, Suo T, Pan H et al. ClZ1 promoted the growth and migration of gallbladder cancer cells. Tumour Biol 2015; 36: 2583-2591.

67 Huang Y, Du Q, Wu W, She F, Chen Y. Rescued expression of WIF-1 in gallbladder cancer inhibits tumor growth and induces tumor cell apoptosis with altered expression of proteins. Mol Med Rep 2016; 14: 2573-2581.

68 Zhang J, Tian XJ, Xing J. Signal transduction pathways of EMT induced by TGFbeta, SHH, and WNT and their crosstalks. J Clin Med 2016; 5: E41.

$69 \mathrm{Li} \mathrm{J,} \mathrm{Wu} \mathrm{T,} \mathrm{Lu} \mathrm{J,} \mathrm{Cao} \mathrm{Y,} \mathrm{Song} \mathrm{N,} \mathrm{Yang} \mathrm{T} \mathrm{et} \mathrm{al.} \mathrm{Immunohistochemical} \mathrm{evidence} \mathrm{of}$ the prognostic value of hedgehog pathway components in primary gallbladder carcinoma. Surg Today 2012; 42: 770-775.

70 Matsushita S, Onishi H, Nakano K, Nagamatsu I, Imaizumi A, Hattori M et al. Hedgehog signaling pathway is a potential therapeutic target for gallbladder cancer. Cancer Sci 2014; 105: 272-280.

$71 \mathrm{Xu} \mathrm{J,} \mathrm{Lamouille} \mathrm{S,} \mathrm{Derynck} \mathrm{R.} \mathrm{TGF-beta-induced} \mathrm{epithelial} \mathrm{to} \mathrm{mesenchymal}$ transition. Cell Res 2009; 19: 156-172.

72 Lamouille S, Xu J, Derynck R. Molecular mechanisms of epithelial-mesenchymal transition. Nat Rev Mol Cell Biol 2014; 15: 178-196.

73 Shu YJ, Weng H, Ye YY, Hu YP, Bao RF, Cao Y et al. SPOCK1 as a potential cancer prognostic marker promotes the proliferation and metastasis of gallbladder cancer cells by activating the PI3K/AKT pathway. Mol Cancer 2015; 14: 12.

74 Kawamoto M, Onishi H, Ozono K, Yamasaki A, Imaizumi A, Nakamura M. TrkB/ BDNF signaling promotes EMT mediated invasiveness and is a potential therapeutic target for gallbladder cancer. Ann Oncol 2016; 27(suppl 6): 701P.

75 Lee DG, Lee SH, Kim JS, Park J, Cho YL, Kim KS et al. Loss of NDRG2 promotes epithelial-mesenchymal transition of gallbladder carcinoma cells through MMP-19-mediated Slug expression. J Hepatol 2015; 63: 1429-1439.

76 Zhang P, Sun Y, Ma L. ZEB1: at the crossroads of epithelial-mesenchymal transition, metastasis and therapy resistance. Cell Cycle 2015; 14: 481-487.

77 Qin Y, Gong W, Zhang M, Wang J, Tang Z, Quan Z. Forkhead box L1 is frequently downregulated in gallbladder cancer and inhibits cell growth through apoptosis induction by mitochondrial dysfunction. PLoS One 2014; 9: e102084.

78 Eferl R, Wagner EF. AP-1: a double-edged sword in tumorigenesis. Nat Rev Cancer 2003; 3: 859-868.

79 Shaulian E. AP-1-the Jun proteins: oncogenes or tumor suppressors in disguise? Cell Signal 2010; 22: 894-899.

80 Lian S, Shao Y, Liu H, He J, Lu W, Zhang Y et al. PDK1 induces JunB, EMT, cell migration and invasion in human gallbladder cancer. Oncotarget 2015; 6: 29076-29086.

81 Shi Y, Lan F, Matson C, Mulligan P, Whetstine JR, Cole PA et al. Histone demethylation mediated by the nuclear amine oxidase homolog LSD1. Cell 2004; 119: 941-953.

82 Shi YJ, Matson C, Lan F, Iwase S, Baba T, Shi Y. Regulation of LSD1 histone demethylase activity by its associated factors. Mol Cell 2005; 19: 857-864.

83 Lin Y, Wu Y, Li J, Dong C, Ye X, Chi Yl et al. The SNAG domain of Snail1 functions as a molecular hook for recruiting lysine-specific demethylase 1. EMBO J 2010; 29: 1803-1816.

84 Lian SX, Shao YB, Liu HB, He JY, Lu WQ, Zhang Y et al. Lysine-specific demethylase 1 promotes tumorigenesis and predicts prognosis in gallbladder cancer. Oncotarget 2015; 6: 33065-33076.

85 Tam WL, Weinberg RA. The epigenetics of epithelial-mesenchymal plasticity in cancer. Nat Med 2013; 19: 1438-1449.

86 von Burstin J, Eser S, Paul MC, Seidler B, Brandl M, Messer M et al. E-cadherin regulates metastasis of pancreatic cancer in vivo and is suppressed by a SNAIL/ HDAC1/HDAC2 repressor complex. Gastroenterology 2009; 137: 361-371, 371.e361-365

87 He J, Shen S, Lu W, Zhou Y, Hou Y, Zhang Y et al. HDAC1 promoted migration and invasion binding with TCF12 by promoting EMT progress in gallbladder cancer. Oncotarget 2016; 7: 32754-32764.

88 Zhang H, Li Y, Lai M. The microRNA network and tumor metastasis. Oncogene 2010; 29: 937-948.

89 Ceppi P, Peter ME. MicroRNAs regulate both epithelial-to-mesenchymal transition and cancer stem cells. Oncogene 2014; 33: 269-278.

$90 \mathrm{Liu} \mathrm{HT}$, Gao P. The roles of microRNAs related with progression and metastasis in human cancers. Tumour Biol 2016; 37: 15383-15397.

91 Hayes J, Peruzzi PP, Lawler S. MicroRNAs in cancer: biomarkers, functions and therapy. Trends Mol Med 2014; 20: 460-469.
92 Chang Y, Liu C, Yang J, Liu G, Feng F, Tang J et al. MiR-20a triggers metastasis of gallbladder carcinoma. J Hepatol 2013; 59: 518-527.

93 Yang G, Zhang L, Li R, Wang L. The role of microRNAs in gallbladder cancer. Mol Clin Oncol 2016; 5: 7-13.

94 Zhang M, Gong W, Zuo B, Chu B, Tang Z, Zhang Y et al. The microRNA miR-33a suppresses IL-6-induced tumor progression by binding Twist in gallbladder cancer. Oncotarget 2016; 7: 78640-78652.

95 Shu YJ, Bao RF, Jiang L, Wang Z, Wang XA, Zhang F et al. MicroRNA-29c-5p suppresses gallbladder carcinoma progression by directly targeting CPEB4 and inhibiting the MAPK pathway. Cell Death Differ 2017; 24: 445-457.

96 Bao RF, Shu YJ, Hu YP, Wang XA, Zhang F, Liang HB et al. miR-101 targeting ZFX suppresses tumor proliferation and metastasis by regulating the MAPK/Erk and Smad pathways in gallbladder carcinoma. Oncotarget 2016; 7: 22339-22354.

97 Lu W, Zhang Y, Zhou L, Wang X, Mu J, Jiang L et al. miR-122 inhibits cancer cell malignancy by targeting PKM2 in gallbladder carcinoma. Tumour Biol 2016; 37: 15615-15625.

98 Ponting CP, Oliver PL, Reik W. Evolution and functions of long noncoding RNAs. Cell 2009; 136: 629-641.

99 Dhamija S, Diederichs S. From junk to master regulators of invasion: IncRNA functions in migration, EMT and metastasis. Int J Cancer 2016; 139: 269-280.

100 Xu Q, Deng F, Qin Y, Zhao Z, Wu Z, Xing Z et al. Long non-coding RNA regulation of epithelial-mesenchymal transition in cancer metastasis. Cell Death Dis 2016; 7: e2254.

101 Brunner AL, Beck AH, Edris B, Sweeney RT, Zhu SX, Li R et al. Transcriptional profiling of long non-coding RNAs and novel transcribed regions across a diverse panel of archived human cancers. Genome Biol 2012; 13: R75.

102 Huarte M. The emerging role of IncRNAs in cancer. Nat Med 2015; 21: 1253-1261.

103 Khandelwal A, Malhotra A, Jain M, Vasquez KM, Jain A. The emerging role of long non-coding RNA in gallbladder cancer pathogenesis. Biochimie 2017; 132: 152-160.

104 Wang SH, Zhang WJ, Wu XC, Zhang MD, Weng MZ, Zhou D et al. Long noncoding RNA Malat1 promotes gallbladder cancer development by acting as a molecular sponge to regulate miR-206. Oncotarget 2016; 7: 37857-37867.

105 Cai Q, Wang Z, Wang S, Weng M, Zhou D, Li C et al. Long non-coding RNA LINC00152 promotes gallbladder cancer metastasis and epithelial-mesenchymal transition by regulating HIF-1alpha via miR-138. Open Biol 2017; 7: 160247.

106 Wang SH, Wu XC, Zhang MD, Weng MZ, Zhou D, Quan ZW. Upregulation of H19 indicates a poor prognosis in gallbladder carcinoma and promotes epithelialmesenchymal transition. Am J Cancer Res 2016; 6: 15-26.

107 Wang SH, Wu XC, Zhang MD, Weng MZ, Zhou D, Quan ZW. Long noncoding RNA $\mathrm{H} 19$ contributes to gallbladder cancer cell proliferation by modulated miR-1945p targeting AKT2. Tumour Biol 2016; 37: 9721-9730.

108 Ma F, Wang SH, Cai Q, Jin LY, Zhou D, Ding J et al. Long non-coding RNA TUG1 promotes cell proliferation and metastasis by negatively regulating miR-300 in gallbladder carcinoma. Biomed Pharmacother 2017; 88: 863-869.

109 Wang SH, Yang Y, Wu XC, Zhang MD, Weng MZ, Zhou D et al. Long non-coding RNA MINCR promotes gallbladder cancer progression through stimulating EZH2 expression. Cancer Lett 2016; 380: 122-133.

110 Ma F, Wang SH, Cai Q, Zhang MD, Yang Y, Ding J. Overexpression of LncRNA AFAP1-AS1 predicts poor prognosis and promotes cells proliferation and invasion in gallbladder cancer. Biomed Pharmacother 2016; 84: 1249-1255.

111 Wang SH, Zhang MD, Wu XC, Weng MZ, Zhou D, Quan ZW. Overexpression of LncRNA-ROR predicts a poor outcome in gallbladder cancer patients and promotes the tumor cells proliferation, migration, and invasion. Tumour Biol 2016; 37: $12867-12875$

112 Lv W, Wang L, Lu J, Mu J, Liu Y, Dong P. Long noncoding RNA KIAA0125 potentiates cell migration and invasion in gallbladder cancer. BioMed Res Int 2015; 2015: 108458

113 Wang L, Zhang Y, Lv W, Lu J, Mu J, Liu Y et al. Long non-coding RNA Linc-ITGB1 knockdown inhibits cell migration and invasion in GBC-SD/M and GBC-SD gallbladder cancer cell lines. Chem Biol Drug Des 2015; 86: 1064-1071.

114 Xiao-Jie L, Ai-Mei G, Li-Juan J, Jiang X. Pseudogene in cancer: real functions and promising signature. J Med Genet 2015; 52: 17-24.

115 An Y, Furber KL, Ji S. Pseudogenes regulate parental gene expression via ceRNA network. J Cell Mol Med 2017; 21: 185-192.

116 Shi X, Nie F, Wang Z, Sun M. Pseudogene-expressed RNAs: a new frontier in cancers. Tumour Biol 2016; 37: 1471-1478.

117 Wu XC, Wang SH, Ou HH, Zhu B, Zhu Y, Zhang $\mathrm{O}$ et al. The NmrA-like family domain containing 1 pseudogene Loc344887 is amplified in gallbladder cancer and promotes epithelial-mesenchymal-transition. Chem Biol Drug Des 2017; 90: 456-463.

118 Lv W, Wang L, Lu J, Mu J, Liu Y, Dong P. Downregulation of TPTE2P1 inhibits migration and invasion of gallbladder cancer cells. Chem Biol Drug Des 2015; 86: 656-662. 
EMT in gallbladder cancer

$\mathrm{S}$ Xu et al

119 Wachter J, Neureiter D, Alinger B, Pichler M, Fuereder J, Oberdanner C et al. Influence of five potential anticancer drugs on wht pathway and cell survival in human biliary tract cancer cells. Int J Biol Sci 2012; 8: 15-29.

120 Yamaguchi J, Sasaki M, Sato Y, Itatsu K, Harada K, Zen Y et al. Histone deacetylase inhibitor (SAHA) and repression of EZH2 synergistically inhibit proliferation of gallbladder carcinoma. Cancer Sci 2010; 101: 355-362.

121 Zhao S, Cao Y, Liu SB, Wang XA, Bao RF, Shu YJ et al. The E545K mutation of PIK3CA promotes gallbladder carcinoma progression through enhanced binding to EGFR. J Exp Clin Cancer Res 2016; 35: 97.
This work is licensed under a Creative Commons Attribution 4.0 International License. The images or other third party material in this
article are included in the article's Creative Commons license, unless indicated otherwise in the credit line; if the material is not included under the Creative Commons license, users will need to obtain permission from the license holder to reproduce the material. To view a copy of this license, visit http://creativecommons.org/licenses/ by/4.0/

(c) The Author(s) 2017 